\title{
Les « tondues » à la Libération :le corps des femmes, enjeu d'une réaproppriation
}

\section{Fabrice Virgili}

\section{OpenEdition}

1 Journals

\section{Édition électronique}

URL : https://journals.openedition.org/clio/518

DOI : $10.4000 /$ clio.518

ISSN : 1777-5299

Éditeur

Belin

Édition imprimée

Date de publication : 1 avril 1995

ISSN : 1252-7017

Référence électronique

Fabrice Virgili, «Les « tondues » à la Libération :le corps des femmes, enjeu d'une réaproppriation », Clio. Femmes, Genre, Histoire [En ligne], 1 | 1995, mis en ligne le 01 janvier 2005, consulté le 22 avril 2022. URL : http://journals.openedition.org/clio/518 ; DOI : https://doi.org/10.4000/clio.518

Ce document a été généré automatiquement le 22 avril 2022

Tous droits réservés 


\title{
Les « tondues » à la Libération :le corps des femmes, enjeu d'une réaproppriation
}

\author{
Fabrice Virgili
}

1 En cette année de commémoration du cinquantenaire de la Libération, force est de constater que la «tondue » reste une des images fortes de cette période. Image forte, mais qui revêt à la fois un caractère honteux - comment expliquer l'absence de «tondue » dans l'exposition de la Mairie de Paris, ou dans le numéro spécial du Parisien Libéré ${ }^{-}$- et simplificateur : « celles qui paient leurs coupables amours des années noires [...] sont tondues publiquement puis promenées dans les rues $»^{2}$. On en reste ainsi, cinquante ans après, à une explication des plus sommaires et surtout incomplète. Car si les tondues sont restées trop longtemps dans ce qu'Alain Brossat ${ }^{3}$ appelle très justement un angle mort de la recherche historique, l'étude des différentes archives ${ }^{4}$ offre aujourd'hui une vision beaucoup plus complexe et variée de ce que fut «la tonte ».

2 Un phénomène massif

3 Que savons nous aujourd'hui sur les tontes? L'état actuel de la recherche ${ }^{5}$ ne nous permet pas de chiffrer précisément ce phénomène. Il n'en demeure pas moins qu'il fut massif. Il concerne toutes les régions de France. Même dans l'Est de la France, que l'on croyait épargné, il y eut « des femmes aux cheveux coupés »; c'est le cas par exemple à Rambervilliers ${ }^{6}$ où des manifestants installent dans un café un «bureau de tonte ", devant lequel passent douze femmes, travailleuses volontaires en Allemagne ou collaboratrices, les 31 mai et 1er juin 1945.

4 Les tontes se déroulent autant dans les grandes villes, qui ont toutes «leurs tondues », qu'en zone rurale. En Charente Inférieure ${ }^{7}$, ce sont les gamins d'un petit village qui à l'exemple de leurs aînés « jouent au maquis... Armés de sabres de bois ils s'emparent du verger, pénètrent au poulailler et libèrent les lapins... Puis tondent trois petites filles ». Plus généralement, les procès verbaux de Gendarmerie offrent, quand ils existent, de nombreux exemples de tontes se déroulant dans des villages; la promenade qui 
accompagne souvent la tonte s'étend alors d'un hameau à l'autre. Ces mentions sont trop nombreuses pour n'être que le fruit du hasard ; on en retrouve pour l'instant dans soixante-dix-sept départements. C'est bien l'importance de cette pratique qui explique la « postérité » des tontes.

5 On imagine aussi trop souvent les tontes comme accompagnant les seules journées de la Libération. Elles commencent en réalité plusieurs mois auparavant et ont été annoncées par certains organes de la presse clandestine ${ }^{8}$. Dès mars 1944, on trouve des mentions concernant des départements aussi éloignés que la Loire-Inférieure et l'Isère. Elles se déroulent alors de manière discrète, le plus souvent de nuit lors d'opérations visant des collaborateurs. Une fois les cortèges de tondues de la Libération passés, cette pratique se poursuit inégalement selon les lieux, sans que l'on sache encore très bien pourquoi elle perdure dans certaines villes. Ainsi, au sujet de quatre ou cinq femmes tondues fin septembre à Tournon, le journal local des FTP9 signale « qu'il n'est jamais trop tard pour bien faire ». Dans une petite ville de l'Oise, c'est une affiche ${ }^{10}$ intitulée « Liste des femmes dites Poules à Boches, n'ayant pas eu les cheveux coupés » qui tente de relancer les tontes début octobre 1944.

6 Une deuxième vague, en revanche, se dessine nettement en mai-juin 1945. Elle correspond à la conjonction de trois phénomènes. C'est la période du retour des déportés, des prisonniers de guerre, des requis au STO, mais aussi des travailleurs volontaires et de celles et ceux qui sont partis avec les Allemands lors de leur retraite. C'est aussi la découverte de l'horreur des camps. Retour des rescapés, images des camps, témoignages publiés par la presse provoquent un véritable choc dans la population ${ }^{11}$. La volonté d'une épuration en profondeur est ainsi relancée. C'est aussi le moment où un certain nombre de personnes, arrêtées à la Libération, sont relâchées après quelques mois d'internement. Pour beaucoup, elles semblent s'en tirer à trop bon compte. Tontes, attentats, exécutions viennent compléter une épuration légale jugée trop clémente ou incomplète par certains. Le Préfet du Jura note dans son rapport bimensuel ${ }^{12}$ :

$C^{\prime}$ est la première fois depuis de très nombreux mois, et dans différents centres du département, on s'est emparé de certaines femmes pour leur couper les cheveux; on s'en prend aussi bien à des femmes de mœurs légères, qu'à d'autres personnes de conditions sociales plus élevées, qui s'étaient fait remarquer pendant l'occupation.

7 On assiste ainsi à ce que l'on pourrait qualifier d' «épuration extra-judiciaire rampante » jusqu'à la fin 1945, voire le début de l'année 1946.

8 Le prolongement des tontes dans le temps ainsi que leur extension sur l'ensemble du territoire expliquent la grande variété de leur déroulement. Beaucoup en effet ne correspondent pas à l'image qui demeure aujourd'hui. La tonte ne se déroule pas toujours devant une foule en liesse ou en furie, elle n'est pas seulement l'œuvre de résistants de la dernière heure, elle ne châtie pas uniquement les relations sexuelles avec l'occupant, et n'est pas toujours un moyen de canaliser la violence vers le lampiste aux dépens des collaborateurs plus importants.

9 Et pourtant c'est bien cette image que la mémoire a conservée ou, pourrions-nous dire, a créée. Les photographies des tontes - fréquemment publiées mais somme toute peu nombreuses ${ }^{13}$-, les romans et quelques récits de témoins sont la partie émergée de cette histoire. Est mis en avant le châtiment : la coupe des cheveux, qui prend le dessus sur le délit. La lecture du châtiment suffit alors à caractériser la "tondue », elle est 
punie dans son corps, c'est donc son corps qui est coupable. La destruction d'un des attributs de la séduction - la chevelure - implique ce que les contemporains appellent la «collaboration horizontale». Les principales intéressées se sont tues et se taisent encore. Les historiens ne se sont pas arrêtés, au-delà de quelques lignes, à ce qui apparaissait peut-être trop comme une anecdote, un épiphénomène, un « décor » de la Libération. D'où l'importance de la mise en perspective de cette image de la "tondue » avec les sources aujourd'hui étudiées.

Ces sources, en fonction de leur origine, offrent une vision partielle et partiale de la tonte. Les mises en scène du corps de la tondue qu'elles décrivent peuvent être résumées à trois fonctions distinctes. Ce corps est, successivement ou simultanément selon les cas, image de la faute, image de châtiment et, ce qui n'est pas le moindre des paradoxes, image positive d'une reconstruction.

La faute

12 Dans les documents étudiés, la faute, ou le crime imputé à la « tondue » occupe souvent une place plus importante que la coupe des cheveux elle-même. Le traumatisme de l'Occupation, les restrictions, les peurs, la faim et toutes les frustrations de la période, semblent alors exploser dans la description de celles qui seraient passées au travers de ces privations. «La vie de noces » supposée de ces femmes apparaît comme une injure aux souffrances du plus grand nombre. Les reproches invoqués peuvent alors toucher chaque aspect de la vie quotidienne : ce sont des meubles et un poste de TSF que l'on reproche à une infirmière de Rochefort-sur-mer ${ }^{14}$ d'avoir obtenu d'un Allemand, comme d'être raccompagnée en voiture, de pouvoir rentrer après l'heure du couvrefeu, de consommer du vin et des liqueurs, d'écouter de la musique et de danser alors que les bals sont interdits, de confectionner des gâteaux pour toutes les autres... La liste de ces griefs est longue.

13 Si l'on a ainsi une image en négatif des frustrations de la population, ce qui exprime le plus ce reproche d'une vie de jouissance dans une période de souffrance est bien sûr l'accusation "d'avoir couché avec les boches". Il y a ainsi, par le vocabulaire de désignation de ces femmes, par la description plus ou moins fantasmée de leurs relations avec les Allemands, la construction d'une image érotisée des « tondues ». C'est probablement un des éléments qui fait encore croire que la tonte est le châtiment exclusif de ces relations sexuelles avec l'ennemi. Les articles de presse, malgré la violence de certains propos tels que "paillasse à boches " ${ }^{15}$, restent dans l'ensemble relativement pudiques. Le vocabulaire est plus feutré, moins directement vulgaire; ainsi le terme le plus fréquemment utilisé est celui de prostituée, accompagné parfois de variations sur le même thème, telles que "égéries à doryphores", "cocodettes frivoles », « hétaïres de haute volée » ou celles qui ont «fridolinisé sur les matelas ». On imagine cependant mal une foule utilisant ces expressions à l'encontre d'un cortège de femmes tondues. Ces expressions "journalistiques» reflètent néanmoins, en les déformant, les sentiments exprimés de manière beaucoup plus directe lors des témoignages recueillis par les gendarmes. On a alors toute une palette de cette rancœur, souvent investie de fantasmes à l'encontre de celles qui sont soupçonnées d'avoir pratiqué « la collaboration horizontale».

L'extrait ci-dessous d'un procès-verbal de gendarmerie montre très clairement la place de la rumeur dans le processus d'accusation d'une femme :

Enquête suite à lettre anonyme dénonçant un avortement de Mme X, 25 ans, ménagère, mari prisonnier de guerre, internée. 
Témoin $n^{\circ} 1$ : «elle est réputée comme étant de mœurs légères et a beaucoup fréquenté les Allemands ».

Témoin $\mathrm{n}^{\circ} 2$ : «cependant à en croire la rumeur publique elle aurait fait un avortement. Il est notoire qu'elle a beaucoup fréquenté les Allemands et qu'elle a été bien critiquée à ce sujet ».

Témoin $n^{\circ} 3$ : «tout ce que je puis dire c'est qu'elle a fréquenté les troupes occupantes ».

Témoin $\mathrm{n}^{\circ} 4:$ : la rumeur publique lui reproche d'avoir collaboré intimement avec les troupes d'occupation ».

Témoin $n^{\circ} 5:$ : à en croire la rumeur publique elle passait pour être enceinte».

Mme $\mathrm{X}:$ « je nie énergiquement les faits qui me sont reprochés » ${ }^{16}$. peu d'importance dans ce cas. On note que la multitude des témoignages à charge n'apporte, ici, pas plus de faits tangibles, ils jouent tous sur un même registre : celui de la réputation de cette femme. Nombreux sont ceux qui ignorent encore l'horreur des camps, les rafles et persécutions de tout ordre, mais beaucoup semblent tout connaître des pratiques sexuelles de l'occupant avec les «filles du pays ». La « rumeur publique " permet de condamner les mœurs par trop légères de ces femmes. Elle a aussi pour fonction de pénétrer les lieux clos, comme le domicile de ces deux Grenobloises où se déroulaient «des noces crapuleuses dont les échos retentissaient dans tout le quartier $\aleph^{17}$. L'observation des allées et venues, l'écoute des bruits d'orgie ou plus simplement de musique, les scènes furtives volées au travers d'une persienne, d'une porte, deviennent ainsi un véritable récit construit sur le réel, l'imaginaire et le fantasme. Il permet au public de faire irruption dans le privé pour une narration de cette «vie de débauche $»^{18}$. La rumeur s'enrichit ainsi de multiples images de la jouissance.

ltivateur de l'Yonne se souvient de « l'avoir vue (l'institutrice du village) donner un soir des cours de français à un officier allemand sur les genoux duquel sa sœur était assise. Ceci se passait chez le marchand de chaussures $»^{19}$. De son côté, une couturière d'un village du Var déclare :

je les ai vues (trois jeunes institutrices tondues dans le village) s'amuser, rire, plaisanter et même un certain jour avec eux (des officiers allemands) jouer à s'arroser en tenue de bain, dans la cour de l'école ${ }^{20}$.

Nombreuses sont ces images qui insistent sur une tenue, une attitude. Ajoutées les unes aux autres, elles offrent une vision parfaite et globale "d'une vie de débauche », jusqu'à ce bûcheron de l'Oise qui déclare aux gendarmes venus l'interroger :

En 1940 j'ai pris des photographies au moment où Mme X se trouvait avec ces militaires dans le jardin du débit, dans des poses qui indiqueraient la mauvaise conduite de cette jeune fille. Je ne peux pas vous les montrer, je les ai cachées en 40 et depuis n'ai pu remettre la main dessus ${ }^{21}$.

Hélène Eck souligne que "la Libération révèle à quel point les circonstances de l'Occupation ont brouillé les frontières de la vie privée et publique $»^{22}$. Toutes ces descriptions en effet dépassent le cadre du simple ragot de voisinage ${ }^{23}$ désignant «l'aguicheuse » ou «la putain du village». Vient s'y superposer un discours d'ordre politique qui, lui, est propre à la période. La «femme de mauvaise vie » l'est avec les Allemands, il ne s'agit plus simplement d'une attitude trop indépendante, d'une sexualité extra-conjugale interdite aux femmes, mais bien d'une trahison. "Cette femme a été prise en flagrant délit d'adultère avec les ennemis de notre Nation $»^{24}$. Morale et politique se confondent alors pour déposséder les femmes de leur propre 
corps. Le châtiment d'une collaboration d'un caractère particulier nécessite de s'appuyer sur le délit d'adultère étendu du cadre familial au cadre national.

Si les tontes sont dans leur quasi-totalité extra-judiciaires, il n'en existe pas moins un débat sur les poursuites pour "collaboration horizontale». Comme le montre ce rapport du Commissaire Régional de la République, les avis sont partagés sur les bases juridiques qui doivent permettre de sanctionner celles qui en sont coupables:

Des divergences se sont produites entre les décisions des Chambres civiques relativement à la répression de la collaboration horizontale. Alors que certaines Chambres civiques se saisissent de tous les cas, quelles que soient les intéressées et les circonstances, certaines autres entendent faire des discriminations. Les unes refusent de prononcer l'Indignité nationale des femmes qui font de la prostitution leur métier, estimant que leur conduite revêt un caractère professionnel et nullement politique. D'autres se demandent si la Chambre civique peut se saisir en l'absence d'une plainte déposée par le mari, la collaboration horizontale étant d'abord un adultère ${ }^{25}$.

Bien qu'il s'agisse là d'interrogations juridiques qui n'ont pas d'effet sur la décision de tondre, elles marquent les réticences à confondre vie privée et vie publique. La référence juridique reste pourtant l'article 1 de l'ordonnance du 26 décembre 44 , qui déclare «coupable d'Indignité nationale tout individu qui a sciemment apporté, en France ou à l'étranger, une aide directe ou indirecte à l'Allemagne». Les relations intimes en font donc partie. La situation des femmes de prisonniers de guerre est encore plus claire.

21 Les sources faisant état de tontes signalent fréquemment un mari prisonnier. Dans l'Oise, le Préfet propose, dans son rapport concernant l'assistance aux prisonniers, " que les procédures soient plus rapides pour donner satisfaction aux rapatriés pouvant apporter des preuves absolument certaines d'inconduite notoire $»^{26}$. On note, pour les femmes de prisonniers, une vigilance accrue du voisinage, une responsabilité collective qui ne leur pardonne pas d'avoir eu des relations avec un autre homme, encore moins si celui ci est membre des troupes d'occupation. La particularité réside dans la complémentarité entre cette surveillance de la communauté et une loi promulguée le 23 décembre 1942 par Vichy - et non supprimée depuis - qui permet «au Ministère Public d'intervenir, sans plainte du mari, pour sanctionner le concubinage notoire d'une épouse d'une personne retenue au loin par suite des circonstances de la guerre ». Pour Michèle Bordeaux ${ }^{27}$, « l'ordre familial est une affaire d'État qui ne peut être confié au seul mari, le Parquet est le substitut du chef de famille ${ }^{28}$.

Il existe bien une représentation sexuelle de la collaboration. Le corps féminin est l'objet de cette trahison, c'est donc ce corps qui doit être châtié.

La tondeuse épuratoire

"Quand la tondeuse vengeresse la privera-t-elle d'un de ses moyens de séduction?" s'interroge l'éditorialiste de La Libération de l'Aunis et de la Saintonge ${ }^{29}$. On assiste alors à une mise en scène du corps de la femme qui a séduit l'ennemi, qui a profité de l'Occupation pour échapper aux souffrances, qui s'est vendu au «boche». Que se soit par les insultes de la foule (« Puisque tu as fait la putain avec eux depuis quatre ans, toi aussi tu vas prendre $»^{30}$ ), l'apposition de pancartes ( raous... j'ai couché avec les

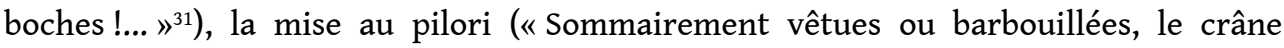
tondu, celles-là passent au pilori avant d'être dirigées sur les prisons ${ }^{32}$ ), ou dans certains cas la dénudation, le corps est mis en avant dans cette cérémonie expiatoire. Le corps est ainsi dégradé par la coupe des cheveux, mais aussi par les coups, les 
inscriptions de croix gammées faites au goudron ou à la peinture, ou encore, en détournant un autre élément de la séduction, le rouge à lèvres ${ }^{33}$. Il s'agit par la tonte non seulement d'exclure la femme de la communauté nationale, mais aussi de détruire l'image de sa féminité. À l'érotisation qui prépare la tonte, succède ainsi un processus de désexualisation. Le corps ne doit être alors que le support des signes de la trahison.

Elles porteront sur leur corps la trace de leur infamie [...] celles qui sont indignes des noms de femme et de Française ${ }^{34}$.

Les coupables perdent leur nom de femme pour n'être plus désignée que sous le vocable de «tondue »; il y a destruction symbolique du corps sexué, la destruction réelle du corps par l'exécution n'étant que très rarement l'issue d'une tonte ${ }^{35}$. Le seul témoignage de la séduction passée de ces femmes réside dans les mentions des « mèches blondes et brunes qui ne tardèrent pas à joncher le sol » ${ }^{36}$. Ces mèches restent sur le sol, la « tondue » s'éloigne, la rupture a bien eu lieu avec les années noires.

La coupe des cheveux a bien pour fonction d'enlaidir ces femmes au point de les « effacer » de la communauté :

Elles portent publiquement les marques de leur infamie [...] elles sont rejetées du sein de la Nation française ${ }^{37}$.

La laideur physique de ces crânes rasés vient naturellement orner ou plutôt révéler à tous leur laideur morale. On relève dans le quotidien Voies Nouvelles ce passage qui semble se complaire dans la description de cette dégradation :

Un être étrange bizarrement humain menait la danse. À force d'écarquiller les yeux, on reconnut des formes féminines et, sous un crâne en boule d'ivoire marqué de la peinture infamante, des yeux torves, une bouche baveuse: la hideur d'un

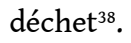

28 Ces descriptions, comprenons-le bien, concernent avant tout les tondues et non les tontes. Celles-ci paraissent bien anodines pour la plupart, quand elles sont mises en parallèle avec les horreurs de l'Occupation et du nazisme. Il s'agit donc de détruire l'image de ces femmes sans détruire l'image d'un peuple qui se libère. Accusation que l'on retrouve cependant dans certaines prises de position: "ne salissons pas notre victoire, notre belle victoire populaire $»^{39}$ proclame par exemple La Marseillaise au sujet d'une dénudation publique. La plupart de ces descriptions jouent plus sur le registre de la moquerie que sur celui de l'horreur. Ce sont des variations sur le thème de "l'esthétique de la nouvelle ondulation ${ }^{40}$ qui sont utilisées dans ces représentations. On ironise ainsi sur ceux « qui ont manié la tondeuse sans se soucier des règles posées par la mode ou l'élégance... Des profanes dans l'art de la peinture (qui ont) employé le goudron pour corriger des femmes de leur inconduite notoire $»^{41}$. Ce jeu d'équilibre entre description de la laideur et préservation de l'image de la Libération conduit au paradoxe de voir les symboles honnis du nazisme devenir «de magnifiques croix gammées ${ }^{{ }^{42}}$ quand celles-ci ornent les joues, le front et la tête des tondues d'Albi.

Les crânes rasés des « collaboratrices horizontales » deviennent une image positive de l'épuration et de la reconstruction et la «tondeuse épuratoire » en est un instrument privilégié. La coupe des cheveux se transforme en mesure d'hygiène, la condition nécessaire au nettoyage du pays.

Il faut qu'à leur retour (prisonniers et déportés) la « désinfection » soit terminée, pour les recevoir dans une Saintonge calmée et propre ${ }^{43}$. femmes renferment « les miasmes de l'infection bochisante $»^{44}$. Au-delà du discours, on 
assiste à une véritable campagne prophylactique dans le département des PyrénéesOrientales où le CDL y prend la mesure suivante :

à l'exception des prostituées des maisons publiques, les femmes qui ont eu des rapports intimes avec les Allemands devront avoir la tête rasée. Et seront en outre soumises pendant six mois à la visite médicale bi-hebdomadaire à laquelle sont astreintes les prostituées surveillées ${ }^{45}$.

La «collaboration horizontale » est vécue, dans le prolongement de l'adultère à la Nation, comme une véritable souillure dont est victime le pays. C'est le corps de Marianne qui en est à la fois l'auteur et la victime. Un avocat, dans une forme de justice particulière aux Cours martiales, peut ainsi réclamer « une punition de rigueur (pour sa cliente, coupable) d'avoir déshonoré la femme française $\aleph^{46}$. La coupe des cheveux doit permettre au pays de retrouver son honneur, d'effacer la souillure portée par le corps de ses femmes. Alain Brossat ${ }^{47}$ l'indique dans un chapitre où il fait le parallèle avec le châtiment des sorcières :

Tout se passe comme si la tondue était chargée d'emporter avec elle dans le désert de l'exil social tous les péchés, tous les crimes de la collaboration.

$C^{\prime}$ est à cette condition que le pays peut retrouver son unité. La tondue devient ainsi un formidable enjeu de réappropriation. La participation active ou passive d'une part importante de la population, la mise en scène du cortège et du châtiment font partie de la reconquête d'un espace perdu. Ainsi comme le souligne Pierre Laborie :

C'est à la lumière de ce passage brutal de l'abattement à l'explosion qu'il faut aussi juger les débordements et les démonstrations excessives de la Libération ${ }^{48}$.

Les tontes - avec les défilés, les maisons pavoisées, les bals - "sont belles» parce qu'elles expriment les promesses de lendemains qui chantent, une fierté retrouvée aux dépens de ces femmes qui n'ont pas compris que, plus que jamais, leur corps ne leur appartient pas. Il est comme tout le reste un enjeu politique. Avoir eu des relations sexuelles avec un soldat allemand devient alors « la grande trahison des garces $»^{49}$.

\section{NOTES}

Absence d'autant plus surprenante que, cinquante ans auparavant dans son cinquième numéro du 26 août 1944, ce même journal publiait sans aucun commentaire une photo de tondue.

2 Paris Match, numéro spécial « La France Libérée », 1994, p. 112. 
Alain Brossat, Les tondues : un carnaval moche, Paris, Manya 1992. Premier ouvrage consacré aux « tondues ", à partir d'un certain nombre de cas et sans prétendre à une quelconque exhaustivité. Alain Brossat aborde le sujet de manière multiforme : en s'arrêtant longuement sur les images de «tondues ", de Duras à Capa en passant par Éluard et Brassens ; en soulignant les ambiguïtés de la relation entre histoire et mémoire ou les similitudes avec d'autres pratiques antérieures : «Grande Peur », charivari, buchers de l'inquisition, et bien sûr carnaval... moche.

\begin{tabular}{|l|l|}
\hline 4 & $\begin{array}{l}\text { Contrairement à ce que l'on pouvait craindre, les archives concernant les tontes à la } \\
\text { Libération sont nombreuses et variées. Aux Archives Nationales, la série } 72 \text { AJ et les } \\
\text { rapports des Commissaires Régionaux de la République et des Préfets (cote F1a 4021/4028, } \\
\text { et F1c 1205/1233) offrent un certain nombre de mentions, essentiellement pour l'année } \\
1945 .\end{array}$ \\
\hline $\begin{array}{l}\text { Aux Archives de la Gendarmerie Nationale, les registres de correspondance et surtout les } \\
\text { procès verbaux établis par les Brigades Territoriales constituent, quand ils existent encore, } \\
\text { des sources d'une grande richesse, avec deux remarques néanmoins : une sur- } \\
\text { représentation du milieu rural ; et des mentions à la condition que les femmes tondues } \\
\text { aient porté plainte, ou qu'elles soient (ou un membre de leur famille) interrogées ou } \\
\text { poursuivies pour « agissements antinationaux » ou tout autre acte de collaboration. }\end{array}$ \\
\hline $\begin{array}{l}\text { Aux Archives Départementales de l'Oise, les dossiers de Commissions d'épuration, des } \\
\text { Chambres civiques et des Cours de justice, avec là aussi la nécessité que les femmes tondues } \\
\text { soient poursuivies devant la justice pour qu'il y ait une mention de la coupe de cheveux. } \\
\text { L'Oise n'est que le premier d'une dizaine de départements les plus représentatifs de la } \\
\text { diversité de l'époque (présence forte du maquis, libération par les Alliés ou la Résistance, } \\
\text { date de la libération, « dureté » de l'épuration en général, régions rurales ou industrielles et } \\
\text { urbaines, etc.), toutes choses qui restent à étudier. }\end{array}$
\end{tabular}

Les sources (voir ci-dessus) étudiées dans le cadre de ma thèse de doctorat, sous la direction de Pierre Laborie à l'Université de Toulouse-Le Mirail, prolongent le dépouillement systématique de la presse de la Libération effectué pour mon DEA, Tontes et tondues à travers la presse de la Libération sous la direction de messieurs Antoine Prost et Jean-Louis Robert, Paris 1-La Sorbonne, 1992 (consultable à l'IHTP).

\begin{tabular}{|l|l|}
\hline 6 & Archives Nationales, 72 AJ 384. \\
\hline
\end{tabular}

\begin{tabular}{l|l}
7 & La Libération de l'Aunis et de la Saintonge, 14 octobre 1944, Saintes.
\end{tabular}

\begin{tabular}{|l|l|}
8 & C'est le cas du premier numéro des Femmes Françaises paru en janvier 1944.
\end{tabular}

$9 \quad$ L'Assaut, 29 septembre 1944, Tournon (Ardèche).

${ }_{10} \quad$ CAGN (Centre Administratif de la Gendarmerie Nationale)-Cie de l'Oise.

11 Anise Postel-Vinay, Jacques Prévotat, «La déportation », in La France des années noires, sous la direction de Jean-Pierre Azéma et François Bédarida, tome 2, Paris, Seuil, 1993. 
$12 \quad$ Archives Nationales F1cIII/1219.

Parmi ces photos, deux reviennent régulièrement : celle de Capa à Chartres, et une autre

13 anonyme faite à Paris dans la cour de la Préfecture, où une femme tondue et tuméfiée tient une pancarte : «A fait fusiller son mari ».

$14 \quad$ CAGN - Cie Charente-Inférieure

$15 \quad$ L'Yonne Républicaine, 19 septembre 1944, Auxerre.

16 CAGN - Cie de Charente-Inférieure.

$17 \quad$ Les Allobroges, 13 septembre 1944, Grenoble.

18 CAGN - Cie de Charente-Inférieure.

$19 \quad$ CAGN - Cie de l'Yonne.

$20 \quad$ Cité par François Rouquet, L'épuration dans l'administration française, Éditions du CNRS, Paris 1994, p. 131.

$21 \quad$ Archives Départementales de l'Oise.

22 Hélène Eck, « Les Françaises sous Vichy », in Histoire des femmes, t. 5, sous la direction de Françoise Thébaud, Paris, Plon, 1992.

${ }^{23} \quad$ On pense par exemple à La fiancée du pirate de Nelly Kaplan.

${ }_{24} \quad$ La Voix de l'Ouest, 28 septembre 1944, Rennes.

Archives Nationales, F1a 4026, rapport du Commissaire Régional de la République de Poitiers. Si de plusieurs régions de France proviennent des interrogations sur le cadre juridique de la répression de la « collaboration horizontale », il n'y a pas réellement de suite au niveau gouvernemental.

$26 \quad$ Archives de l'Oise 33W 8270.

Michèle Bordeaux, « Sept ans de réflexion, divorce et ordre social (1940-1945) », dans Droit Histoire et Sexualité, textes réunis par Jacques Poumarède et Jean-Pierre Royer, Paris, L'espace juridique, 1987, pp. 229-247.

${ }_{28} \quad$ Ce que semble ignorer les Chambres civiques de la Vienne; voir supra. 
\begin{tabular}{l|l}
29 & La Libération de l'Aunis et de la Saintonge, 15 septembre 1944, Saintes.
\end{tabular}

\begin{tabular}{l|l}
30 & CAGN - Cie de l'Oise.
\end{tabular}

${ }_{31} \quad$ L'Assaut, 4 septembre 1944, Privas.

$32 \quad$ L'Aube Libre, 5 septembre 1944, Troyes.

3 Ce Soir, 24 août 1944, Paris.

$34 \quad$ L'Écho de la Corrèze, 14 septembre 1944, Tulle.

Quelques femmes ont été abattues ou fusillées après avoir été tondues. Ces cas sont rares, mais avec l'exemple de celles qui sont tondues puis internées, montrent bien que la tonte n'est substitutive d'aucun autre châtiment.

${ }_{36} \quad$ L'Assaut, 25 septembre 1944, Privas.

${ }^{37} \quad$ La Marseillaise, 25 août 1944, Marseille.

$38 \quad$ Voies Nouvelles, 7 septembre 1944, Périgueux.

39 $\quad$ La Marseillaise, 3 septembre 1944, Marseille.

40 Les Allobroges, 25 août 1944, Grenoble.

\begin{tabular}{|l|l|}
\hline 41 & La Croix du Nord, 8 septembre 1944, Lille.
\end{tabular}

${ }_{42} \quad$ Le Tarn Libre, 29 août 1944, Albi.

43 La Libération de l'Aunis et de la Saintonge, 15 septembre 1944, Saintes.

${ }_{44} \quad$ L'Union Champenoise, 1er septembre 1944, Reims.

${ }_{45} \quad$ La Voix de la patrie, 13 septembre 1944, Perpignan.

46 La France Libre, 19 septembre 1944, Cahors.

\begin{tabular}{|l|l}
${ }_{47}$ & Alain Brossat, op. cit.
\end{tabular} 
${ }_{48} \quad$ Pierre Laborie, L'opinion française sous Vichy, Paris, Seuil, 1990.

\begin{tabular}{|l|l|}
\hline 49 & Les Allobroges, 5 septembre 1944, Grenoble.
\end{tabular}

\section{RÉSUMÉS}

Alors que la «tondue» est une des images fortes de la Libération, les études furent peu nombreuses sur la question.Cette pratique fut pourtant massive et répandue sur l'ensemble du territoire français en 1944-45. Lors de ces tontes, on assiste à une véritable mise en scène du corps de ces femmes. Celles-ci, au-delà de la description d'une pratique de l'épuration extrajudiciaire, sont révélatrices de cette période où les frontières entre vie publique et privée sont largement brouillées. Cette mise en scène ostentatoire laisse alors une grande place au fantasme et à la sexualité. Elle permet de constituer un système de représentation dont les trois facettes sont : la «faute »- la collaboration -, le «châtiment »- l'épuration -, et enfin « une vision de l'avenir» - la reconstruction. Cela permet de mieux comprendre pourquoi ces tontes semblent bien avoir été une " évidence " pour l'époque, et quels ont été les enjeux de cette réappropriation du corps, de la «tondue » en particulier et des femmes en général

Fabrice Virgili : The " tondues » during the Liberation

Altough the "femme tondue» (woman whose head was shaved for alleged collaboration) is a powerful image of the Liberation, few studies have analysed this issue. This practice was common and widespread on French territory in 1944 and 1945. During these headshaving incidents, women's bodies were actually put on display as a means of atonement. Beyond illustrating how thepublic took the law into its own hands, however, the description of « public shaving " reflects during this period how unclear the lines were between public and private life. This theatrical public display opened the way to fantasy and sexuality. Symbolically headshaving eoisodes represented the " $\sin »$ - collaboration -, the " punishment »-purification -, and finally a " vision of the future » - the reconstruction. This study allows us to understand why these episodes occured, as well as the underlying stakes in the reappropriation of the bodies of the "tondue ", specifically, and of women's bodies, in general.

\section{AUTEUR}

\section{FABRICE VIRGILI}

Fabrice VIRGILI, agrégé d'histoire, enseigne dans un collège d'Aubervilliers. Auteur, sous la direction d'Antoine Prost et Jean-Louis Robert, d'un mémoire de DEA Tontes et tondues à travers la presse de la Libération, il poursuit, sous la direction de Pierre Laborie à l'Université de Toulouse-Le Mirail, une thèse de doctorat sur « les tontes à la Libération ». 